\title{
Influence of "J"-Curve Spring Stiffness on Running Speeds of Segmented Legs during High-Speed Locomotion
}

\author{
Runxiao Wang, Wentao Zhao, Shujun Li, and Shunqi Zhang \\ School of Mechanical Engineering, Northwestern Polytechnical University, Xian, Shaanxi 710072, China \\ Correspondence should be addressed to Wentao Zhao; semper_zhaowentao@hotmail.com
}

Received 12 May 2016; Revised 22 September 2016; Accepted 1 November 2016

Academic Editor: Young-Hui Chang

Copyright ( 2016 Runxiao Wang et al. This is an open access article distributed under the Creative Commons Attribution License, which permits unrestricted use, distribution, and reproduction in any medium, provided the original work is properly cited.

\begin{abstract}
Both the linear leg spring model and the two-segment leg model with constant spring stiffness have been broadly used as template models to investigate bouncing gaits for legged robots with compliant legs. In addition to these two models, the other stiffness leg spring models developed using inspiration from biological characteristic have the potential to improve high-speed running capacity of spring-legged robots. In this paper, we investigate the effects of "J"-curve spring stiffness inspired by biological materials on running speeds of segmented legs during high-speed locomotion. Mathematical formulation of the relationship between the virtual leg force and the virtual leg compression is established. When the SLIP model and the two-segment leg model with constant spring stiffness and with "J"-curve spring stiffness have the same dimensionless reference stiffness, the two-segment leg model with "J"-curve spring stiffness reveals that (1) both the largest tolerated range of running speeds and the tolerated maximum running speed are found and (2) at fast running speed from 25 to $40 / 92 \mathrm{~m} \mathrm{~s}^{-1}$ both the tolerated range of landing angle and the stability region are the largest. It is suggested that the two-segment leg model with "J"-curve spring stiffness is more advantageous for high-speed running compared with the SLIP model and with constant spring stiffness.
\end{abstract}

\section{Introduction}

Owing to the elastic elements (muscles, tendons, ligaments, and other soft tissues) of legged systems, in fast animal locomotion spring-like leg behavior is discovered to represent bouncing gaits like running, hopping, and trotting $[1,2]$. On the one hand, the leg compliance can reduce the impact of the ground contact phase [3] and recycle kinetic energy by using of elastic strain energy storage and release [4], which can lead to low energy costs. For instance, due to storage and utilization of elastic strain energy, the reduction rate of the required muscle work is up to 40 percent in a trotting or galloping horse [5]. On the other hand, compliant legged systems exhibiting self-stability in response to internal (speed variations) and external perturbations (ground surface irregularities) can simplify the dynamic control of bouncing motion [6]. Therefore, the elastic elements play an important role in fast animal locomotion [7].

According to spring-like leg behavior of bouncy gaits, biomechanists [8] propose a simple spring-mass model to describe animal and human locomotion, which is also called spring-loaded inverted pendulum (SLIP), consisting of a point mass representing the body and a massless linear spring describing the leg. For the purpose of achieving stable running in the SLIP model, an increase in leg stiffness is required for increased running velocities when angle of attack is unchanged [9]. At present, the SLIP model is extensively applied to the study of bouncing gaits for legged robots, including one-legged hopping robots, bipedal robots, quadruped robots, and hexapod robots. Sato and Buehler propose a hopping robot with one leg based on the SLIP model [10].

However, biological limbs are not the telescopic linear leg model, rather, they are made up of multiple joints; their compliance is situated at the joint level [11,12]. According to this concept, Rummel and Seyfarth [6] study the effects of the two-segment leg model with constant spring stiffness on running stability during low-speed running, and results from simulations and experiments show that adjustment of joint stiffness is required to support stable running at different speeds. Furthermore, this segmented leg supports self-stable running at an enlarged range of speeds (lower minimum 


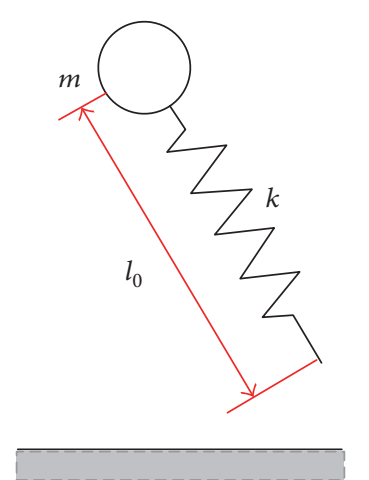

(a) Model parameters

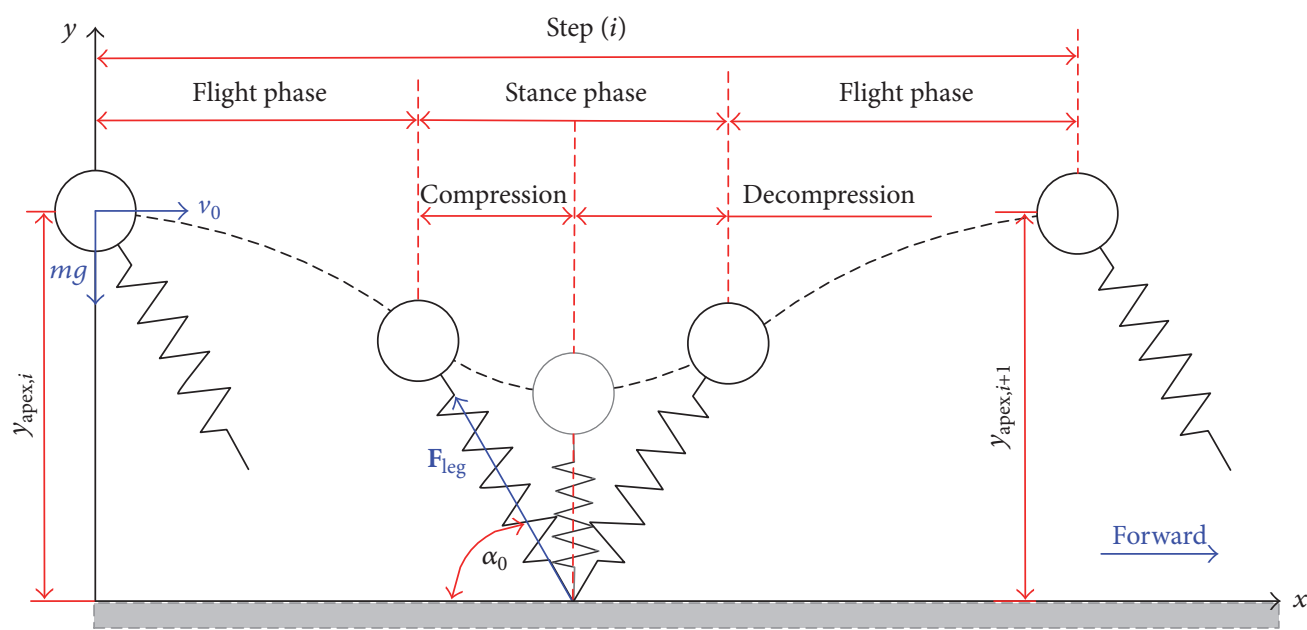

(b) Locomotion phases and transition conditions

FIGURE 1: SLIP model during a running cycle.

speed) compared with the SLIP model. At present, the twosegment leg model with constant spring stiffness is widely applied to the design of compliant legged robots such as the intersegmental joint configuration of "JenaHopper" designed by Rummel et al. [13].

It is well known that the SLIP model and the two-segment leg model with constant spring stiffness can be seen as an effective tool to research on bouncing gaits for legged robots. In addition to these two models, Karssen and Wisse [14] also propose the running model with nonlinear leg spring and study the effect of the nonlinear leg springs on disturbance rejection behavior. Some results of this optimization reveal that the push (push forward and backward) disturbances rejection with the optimal nonlinear leg spring is much better than with the optimal linear spring. What is more, it can be seen from the size of the basin of attraction that the range of running speeds of the optimal nonliner spring is largely increased compared with the optimal linear spring. But this running model is analyzed in the low-speed case. At present, a number of prominent high-speed spring-legged robots, such as bipedal robot MABEL [15] and Cheetah-cub quadruped robot [16], are developed. Here, it is interesting to note that a speed record of $3.06 \mathrm{~m} \mathrm{~s}^{-1}$ is implemented on MABEL by adjusting its effective leg stiffness [17].

Although there are many running robots and running models at present, we focus only on the two-segment leg model in this study. This is because it is the most reduced leg configuration; in spite of the low complexity of this twosegment leg model, it is still suitable to solve the question of how leg segmentation and joint stiffness influence the stability of running at different speeds [6]. On the other hand, so far, the potential effects of joint stiffness on running speeds during high-speed locomotion remain an unresolved issue. Therefore, in the present paper, we propose the twosegment leg model with "J"-curve spring stiffness. Here, "J"curve spring stiffness is inspired by biological materials [18]. Subsequently, we employ this proposed model to investigate the potential role of "J"-curve spring stiffness on running speeds during high-speed locomotion. Here, in this paper running models focus only on the SLIP model, the twosegment leg model with constant spring stiffness and with "J"-curve spring stiffness. This is to develop a deeper understanding of the benefits and drawbacks of the two-segment leg model with "J"-curve spring stiffness compared with the other two models regarding high-speed running capacity.

In this study, we not only hope that the two-segment leg model with "J"-curve spring stiffness will show the largest range of running speed for self-stable high-speed running in all three models but also expect that results of our work will be regarded as a promising concept for the design of bioinspired high-speed robots.

\section{Methods}

2.1. SLIP Model. As shown in Figure 1(a), the SLIP model is modeled as a point mass $m$ attached to a massless spring leg with linear stiffness of $k$ and rest length of $l_{0}$. This model for running can be represented as the flight and stance phases alternatively, which is shown in Figure 1(b). During the flight phase the system dynamics is determined by the point-mass gravity, which results in a ballistic trajectory of the point mass, and then the equation of motion can be expressed as

$$
\mathbf{F}_{\text {flight }}=m \mathbf{g}
$$

where $\mathbf{F}_{\text {flight }}$ is the total force vector during flight and $\mathbf{g}=$ $[0,-g]^{\mathrm{T}}$ denotes the gravitational acceleration vector. In addition, considering that the total energy $E_{\text {total }}$ is assumed to be conserved, during flight $E_{\text {total }}$ is

$$
E_{\text {total }}=\frac{1}{2} m v_{0}^{2}+m g y_{\text {apex }, i},
$$

where $v_{0}$ is the constant horizontal speed of the point mass and $y_{\text {apex }, i}$ denotes the apex height (the point mass has the maximum vertical height at the beginning of the flight phase 


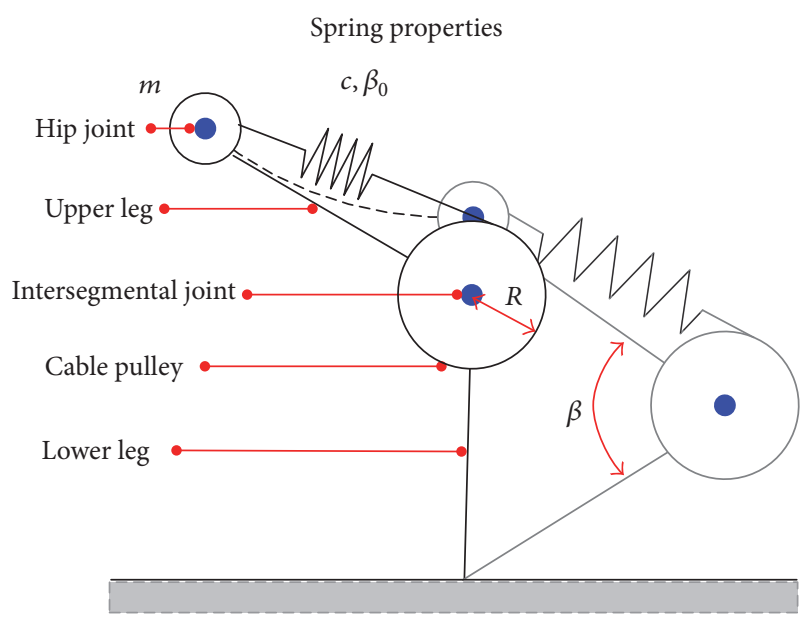

FIgURE 2: The configuration of the two-segment leg model.

of running). Afterwards, the transition from flight to stance phase occurs when the spring leg strikes the ground at a given angle of attack $\alpha_{0}$. This transition event can be formulated by

$$
l(t)=l_{0}
$$

where $l(t)$, which is the function of time $t$, denotes the current spring leg length. The next moment, the stance phase starts. With the tip of foot regarded as pivot without slipping, the stance phase can be split into the compression and decompression subphases. Here, the transition between the above-described two subphases takes place when the leg reaches its maximum compression. Note that leg stiffness is a fixed value of $k$ and the direction of leg force is from the tip of foot to the point mass during these two subphases. Thus, the motion equations of stance phase are written as

$$
\begin{aligned}
\mathbf{F}_{\text {stance }} & =m \mathbf{g}+\mathbf{F}_{\text {leg }} \\
F_{\text {leg }} & =k\left(l_{0}-l(t)\right),
\end{aligned}
$$

where $\mathbf{F}_{\text {leg }}$ is the leg force vector and $\mathbf{F}_{\text {stance }}$ denotes the total force vector. Finally, when the tip of foot leaves the ground the flight phase starts, and then the point mass reaches the subsequent apex variable $y_{\text {apex }, i+1}$. Consequently, a step, called a cycle, can be defined as the movement between $y_{\text {apex }, i}$ and $y_{\text {apex }, i+1}$.

2.2. Two-Segment Leg Model. The following section describes the configuration of the two-segment leg model and its dynamics of running with spring-like legs. As illustrated in Figure 2, the two-segment leg model is described by a point mass $m$ attached to a rotating segmented leg, and this segmented leg is represented by massless upper and lower leg linked by the intersegmental joint, with the joint angle of $\beta_{0}$ at rest and the radius of cable pulley of $R$. What is more, this model can be considered as hip movement actively and knee movement passively. Note that knee joint elasticity originates from spring compliance, and spring property has a significant influence on running stability. Thus, the two-segment leg model can be divided into the two-segment leg model with constant spring stiffness and with nonlinear spring stiffness in terms of the linear or nonlinear characteristics of tensile spring, and its joint stiffness $c(\Delta \beta)$ is

$$
\begin{gathered}
c(\Delta \beta)=\frac{F_{\text {spring }} R}{\Delta \beta} \\
\Delta \beta=\beta_{0}-\beta,
\end{gathered}
$$

where $\beta$ denotes instantaneous joint angle, $\Delta \beta$ represents the amount of joint flexion, and $F_{\text {spring }}$ is the tensile force of the spring. In addition, it is evident that both upper leg of length $l_{1}$ and lower leg of length $l_{2}$ affect the dynamics of running. Therefore, to make the analysis of the mathematical model easily, the two segment lengths are defined as $l_{1}=l_{2}=L$; in order to facilitate the comparison between the SLIP model and the two-segment leg model, rest length of the virtual leg is also defined as $l_{0}$.

Figure 3 illustrates the two-segment leg model during a running period. It is worth noting that the two-segment leg model can be conceived as an equivalent SLIP model whose leg stiffness is nonlinear. Thus, similar to the SLIP model, the two-segment leg model for running is also composed of flight and stance phases, and stance phase can also be divided into the compression and decompression subphases. Similarly, the total energy, transition conditions, the total force during flight, and the direction of leg force of the stance phase are identical with those of the SLIP model, respectively.

2.2.1. Two-Segment Leg Model with Constant Spring Stiffness. As can be seen in Figures 2 and 3, if tensile spring has a constant stiffness, the system can be regarded as the twosegment leg model with constant spring stiffness reported in [6], and the equation of motion for this running system is given by the following equation (based on [6]):

$$
\begin{aligned}
\mathbf{F}_{\text {stance }} & =m \mathbf{g}+\mathbf{F}_{\text {leg }} \\
F_{\text {leg }} & =\frac{\sqrt{l_{1}^{2}+l_{2}^{2}-2 l_{1} l_{2} \cos \beta}}{l_{1} l_{2}} \frac{c\left(\beta-\beta_{0}\right)}{\sin \beta} .
\end{aligned}
$$

2.2.2. Two-Segment Leg Model with "J"-Curve Spring Stiffness. In this section we illustrate a nonlinear "J"-curve spring force-elongation relationship of the proposed model and its dynamics of running. A schematic diagram of a joint of large mammals, presented in [19], describes a knee or an ankle; joint configuration shown in this schematic diagram is similar to the two-segment leg model. Here, owing to the similar role of elastic elements, muscles and tendons shown in this schematic diagram can be considered as tensile spring of the two-segment leg model. it is very interesting to note that muscles [20], tendons [21], and muscle-tendon complex [22] force-elongation curves resemble a "J"-shape, and Steven Vogle also notices that biological tissues (ligaments, skin, etc.) force-length curves resemble a "J"-shape [23]. Thereby, we imitate a joint configuration of large mammals and adopt " $\mathrm{J}$ "curve spring stiffness inspired by biological materials [18] to establish the two-segment leg model with "J"-curve spring 


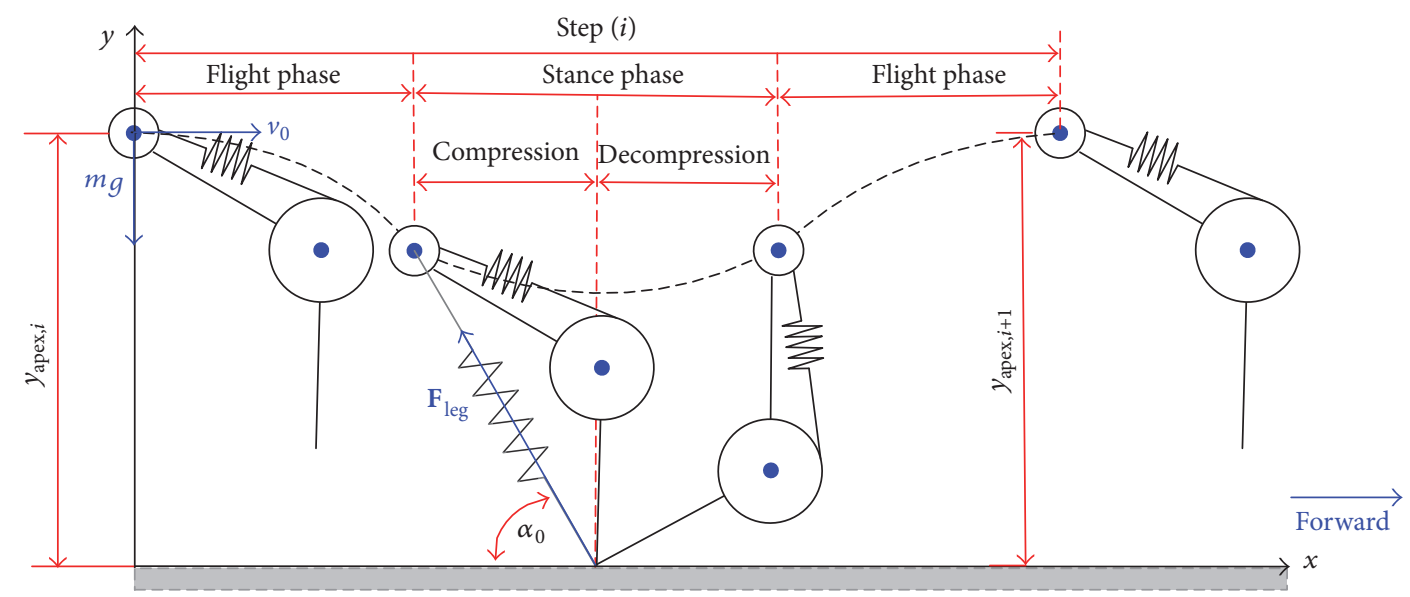

FIGURE 3: Two-segment leg model during a running period.

TABLE 1: Five points representing “J” curve.

\begin{tabular}{lccccc}
\hline Elongation & $0 \%$ & $25 \%$ & $50 \%$ & $75 \%$ & $100 \%$ \\
Force & $0 \%$ & $4 \%$ & $17 \%$ & $48 \%$ & $100 \%$ \\
\hline
\end{tabular}

stiffness. By doing this, we hope that the proposed twosegment leg model with "J"-curve spring stiffness will be capable of realizing high-speed locomotion, resulting in the imitation of biological high-speed running capacity. Here, five points shown in Table 1, presented in [18], can be used to describe "J"-curve spring properties, and then mathematical formulation of the relationship between the "J"-curve spring force $F_{s}\left(l_{s}\right)$ and the "J"-curve spring elongation $l_{s}$ is obtained by using of the interpolation method, and this equation is represented by

$$
F_{s}\left(l_{s}\right)=a_{4}\left(l_{s}\right)^{4}+a_{3}\left(l_{s}\right)^{3}+a_{2}\left(l_{s}\right)^{2}+a_{1} l_{s}+a_{0},
$$

where $a_{4}, a_{3}, a_{2}, a_{1}$, and $a_{0}$ denote the undetermined coefficients; subsequently, substituting these five points into (7) can yield

$$
\begin{aligned}
& a_{4}=-\frac{16 F_{\max }}{25\left(l_{\max }\right)^{4}}, \\
& a_{3}=\frac{48 F_{\max }}{25\left(l_{\max }\right)^{3}}, \\
& a_{2}=-\frac{11 F_{\max }}{25\left(l_{\max }\right)^{2}}, \\
& a_{1}=\frac{4 F_{\max }}{25 l_{\max }} \\
& a_{0}=0,
\end{aligned}
$$

where $l_{\max }$ represents the maximum elongation of "J"-curve spring and $F_{\max }$ denotes the "J"-curve spring force corresponding to $l_{\max }$.
The virtual leg force vector $\mathbf{F}_{\text {leg }}(\Delta \mathbf{l})$ relates gravitational force vector $m \mathbf{g}$ to the total force vector $\mathbf{F}_{\text {stance }}$ during the stance phase with

$$
\mathbf{F}_{\text {stance }}=m \mathbf{g}+\mathbf{F}_{\text {leg }}(\Delta \mathbf{l}) ;
$$

in [6] the virtual leg force $F_{\text {leg }}(\Delta l)$ is a function of the virtual leg compression $\Delta l$ with

$$
F_{\text {leg }}(\Delta l)=\frac{\left(l_{0}-\Delta l\right) \tau}{L^{2} \sin \left(\beta_{0}-\Delta \beta\right)},
$$

where the joint torque $\tau$ can be determined according to $F_{s}\left(l_{s}\right)$ with

$$
\tau=F_{s}\left(l_{s}\right) R
$$

the relationship between the amount of the joint flexion $\Delta \beta$ and $l_{s}$ can be given by

$$
\Delta \beta=\frac{\left(180^{\circ} l_{s}\right)}{(R \pi)}
$$

$\Delta l$ can be then represented by

$$
\Delta l=l_{0}-L \sqrt{2-2 \cos \left(\beta_{0}-\Delta \beta\right)} .
$$

Seeing that the maximum compression of the virtual leg rarely exceeds $30 \%$ of the rest virtual leg length among running animals [24], both the spring's maximum compression in the SLIP model and the maximum compression of the virtual leg in the two-segment leg model are defined as $0.3 l_{0}$ in this study, and then the corresponding maximum amount of the joint flexion $\Delta \beta_{30}$ in the two-segment leg model is formulated by

$$
\Delta \beta_{30}=\beta_{0}-\arccos \frac{2 L^{2}-\left(0.7 l_{0}\right)^{2}}{2 L^{2}} .
$$

Finally, the radius of cable pulley $R$ can be obtained based on the amount of the joint flexion $\Delta \beta_{30}$ with

$$
R=\frac{180^{\circ} l_{\max }}{\pi \Delta \beta_{30}} .
$$


2.3. Analysis Methods. The steps-to-fall analysis and the apex return map, which are reported in $[6,9]$, are adopted to analyze the running dynamics as represented by the abovementioned three models. In the following section, we briefly review these two methods. The steps-to-fall analysis can record the maximum number of steps to fall for a given system parameters $\left(m, l_{0}, v_{0}\right.$, etc.), and simulation calculation is not stopped until the number of predefined steps is reached. Here, the maximum number of successive steps is defined as 50 [6]. Although the steps-to-fall analysis can provide a method for counting the number of successive steps, the system might still fall after a finite threshold. Therefore, in order to solve this question, the apex return map is adopted.

The second approach, the apex return map, can identify the fixed point. Furthermore, the stability of the fixed point $y^{*}=y_{\text {apex }, i+1}=y_{\text {apex }, i}$ can be described by

$$
s=\left.\frac{d y_{\text {apex }, i+1}}{d y_{\text {apex }, i}}\right|_{y^{*}} ;
$$

here if $s$ is smaller than 1 , this condition means that the system is stable.

2.4. Simulation Parameters Setup. For the purpose of facilitating the comparison of all three models, simulation parameters can be defined as follows. (1) Seeing that a representative value for humans running is leg compression at $10 \%$ of rest leg length [25], the reference stiffness $k_{10 \%}$ can be defined by the following equation (based on [6]):

$$
k_{10 \%}=\frac{F_{10 \%}}{l_{10 \%}},
$$

where $l_{10 \%}$ denotes a reference leg compression at $10 \%$ of rest leg length and $F_{10 \%}$ is the corresponding leg force; (2) a dimensionless reference stiffness $\widetilde{k}$ can be expressed by the following equation (based on [26]):

$$
\widetilde{k}=\frac{k l_{0}}{m g} ;
$$

(3) the dimensionless reference stiffness $\widetilde{k}_{10 \%}$ can be given by the following equation (based on [6]):

$$
\widetilde{k}_{10 \%}=\frac{k_{10 \%} l_{0}}{m g} ;
$$

three models parameters, $m=80 \mathrm{~kg}, l_{0}=1 \mathrm{~m}, 30^{\circ} \leq$ $\alpha_{0} \leq 90^{\circ}$, and $0 \leq \widetilde{k}_{10 \%} \leq 50$, and the initial apex height of $1 \mathrm{~m}$ are defined [6]. Additionally, in the two-segment leg models the small $\left(\beta_{0}=115^{\circ}\right)$ and large nominal joint angles $\left(\beta_{0}=165^{\circ}\right)$ are adopted to analyze the effects of different nominal joint angles on running speeds, respectively; in our model $\left(\beta_{0}=115^{\circ}\right.$ and $\left.165^{\circ}\right)$, the maximum speed of stable running is 92 and $40 \mathrm{~m} \mathrm{~s}^{-1}$, respectively. Thus, two speed ranges ( 5 to $92 \mathrm{~m} \mathrm{~s}^{-1}$ and 5 to $40 \mathrm{~m} \mathrm{~s}^{-1}$ ) are utilized to investigate the advantages and disadvantages of our model compared with the other two models with respect to highspeed running capacity, respectively. Here, the speed of $5 \mathrm{~m} \mathrm{~s}^{-1}$ is the minimum speed of stable running in our model at $\beta_{0}=115^{\circ}$.

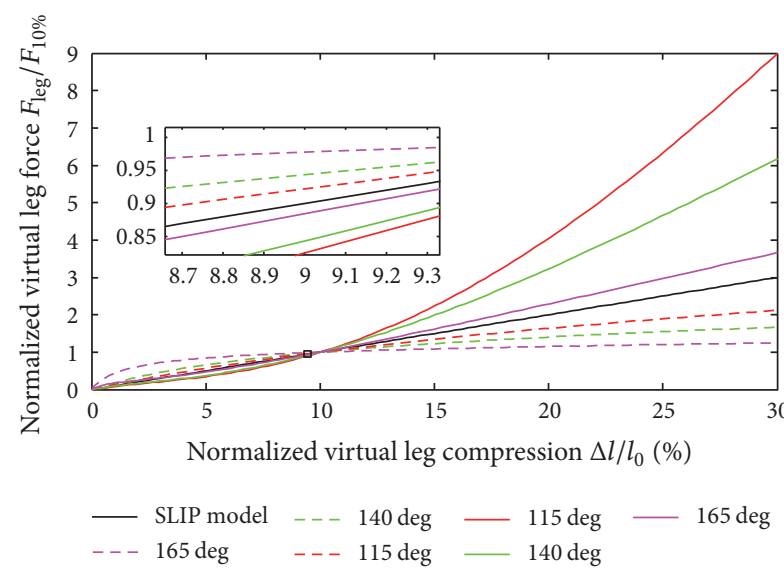

FIGURE 4: Normalized force-compression relationships of the SLIP model and the two-segment leg model. Solid curves are the proposed model and dashed ones denote the two-segment leg model with constant spring stiffness. The same nominal joint angle $\beta_{0}$ is adopted to facilitate the comparison of normalized force-length relationships between the two-segment leg model with "J"-curve spring stiffness and with constant spring stiffness, with the fact that red, green, and purple curves represent $\beta_{0}=115^{\circ}, \beta_{0}=140^{\circ}$, and $\beta_{0}=165^{\circ}$, respectively.

\section{Results}

3.1. Normalized Force-Length Relationships of the TwoSegment Leg Model with "J"-Curve Spring Stiffness. In this section, we not only analyze normalized force-compression relationships of the two-segment leg model with "J"-curve spring stiffness but also investigate the effects of different nominal joint angles $\beta_{0}$ on these relationships. For ease of understanding, we divide the range of $\Delta l / l_{0}$ into two subintervals. Here, the first one is $0 \leqslant \Delta l / l_{0}<10$, and the second is $10 \leqslant \Delta l / l_{0} \leqslant 30$. For $0 \leqslant \Delta l / l_{0}<10$, results shown in Figure 4 reveal that large variations in $\Delta l / l_{0}$ result in small changes in leg force $F_{\text {leg }} / F_{10 \%}$. In contrast, for $10 \leqslant \Delta l / l_{0} \leqslant 30$, large changes in $\Delta l / l_{0}$ lead to large variations in leg force. Furthermore, at the beginning of the second subinterval, a given variations in $\Delta l / l_{0}$ lead to increasingly large changes in leg force with increasing virtual leg compression. Next, we consider roles of nominal joint angles. The larger the nominal joint angle, the slightly smaller the leg force for a given $\Delta l / l_{0}$ in the first subinterval. In the second subinterval, the smaller the nominal joint angle, the greater the rise in leg stiffness at a given virtual leg compression.

3.2. Comparison of Normalized Force-Length Relationships between the Two-Segment Leg Model with "J"-Curve Spring Stiffness and the Other Two Models. Considering that in [6] comparison of normalized force-length relationships between the SLIP model and the two-segment leg model with constant spring stiffness has already been discussed, this section focuses on comparison of relationships between the two-segment leg model with "J"-curve spring stiffness and the other two models. In the first subinterval, leg force of the proposed model is smaller than those of the other two models for a given $\Delta l / l_{0}$, and leg stiffness of our model 
becomes increasingly small with increasing $\beta_{0}$. In the second subinterval, for a given $\Delta l / l_{0}$ our model has the maximum leg force in all three models, and leg force becomes increasingly large with increasing $\beta_{0}$ in our model.

3.3. Stability Analysis at Different Running Speeds $v_{0}$ and Nominal Joint Angles $\beta_{0}$. In this section, we concentrate on the effects of a running speed and the dimensionless reference stiffness on each other. Again, to gain better insights into advantages and disadvantages of our model compared with the other two models regarding high-speed running capacity, we also analyze the effects of these speeds on angle of attack and the stability region in all three models, respectively. Here, for ease of understanding, some typical examples, which are the regions of stable running at different running velocities $\left(v_{0}=7 \mathrm{~m} \mathrm{~s}^{-1}, v_{0}=29 \mathrm{~m} \mathrm{~s}^{-1}\right.$, and $\left.v_{0}=36 \mathrm{~m} \mathrm{~s}^{-1}\right)$ and nominal joint angles $\left(\beta_{0}=115^{\circ}\right.$ and $\left.\beta_{0}=165^{\circ}\right)$, are used to analyze self-stabilizing behavior of the above-represented three models according to the normalized force-compression relationships depicted in Figure 4. Interestingly, the speed of $29 \mathrm{~m} \mathrm{~s}^{-1}$, which is the maximum running speed of the cheetah, is the highest running speed recorded from land animal [27].

In all three models, the proposed model has the tolerated maximum range of the dimensionless reference stiffness at a running speed from 7 to $40 / 92 \mathrm{~m} \mathrm{~s}^{-1}$; the smaller the nominal joint angles, the larger the stability regions for given combinations of $v_{0}$ and $\widetilde{k}_{10 \%}$, as shown in Figure 5(a). In other words, we can see from Figure 5(a) that for a given dimensionless reference stiffness the proposed model exhibits not only the largest tolerated range of running speeds but also the tolerated maximum running speed in all three models; for example, for a given $\widetilde{k}_{10 \%}=41.2698$, our model at $\beta_{0}=115^{\circ}$ is capable of accomplishing stable running behavior at a speed range from 5 to $76 \mathrm{~m} \mathrm{~s}^{-1}$. However, at the same dimensionless reference stiffness, the tolerated speed range is from 5 to $27 \mathrm{~m} \mathrm{~s}^{-1}$ in the SLIP model; worse still, in the two-segment leg model with constant spring stiffness this range is only from 5 to $19 \mathrm{~m} \mathrm{~s}^{-1}$. In addition, a tolerated minimum $\widetilde{k}_{10 \%}$ is required in order to guarantee the stability of running in a region (one of the twelve regions in Figure 6). Interestingly, although the values of these $\widetilde{k}_{10 \%}$ become increasingly large with increasing running speeds, this tolerated minimum $\widetilde{k}_{10 \%}$ in our model is smaller than those of the other two models for a given speed from 7 to $40 / 92 \mathrm{~m} \mathrm{~s}^{-1}$; the smaller the nominal joint angle, the smaller the rate of increment in this tolerated minimum $\widetilde{k}_{10 \%}$.

At fast running speed form 25 to $40 / 92 \mathrm{~m} \mathrm{~s}^{-1}$, our model has the maximum range in angle of attack $\left(\alpha_{1}\right.$ and $\left.\alpha_{2}\right)$. For instance, at high running speed $\left(29 \mathrm{~m} \mathrm{~s}^{-1}\right), \alpha_{1}$ and $\alpha_{2}$ (see Figure 5(b)) are 11.43 and $7.62^{\circ}$ in our model at $\beta_{0}=115^{\circ}$, respectively. In contrast, $\alpha_{1}=\alpha_{2}=3.8^{\circ}$ in the SLIP model and $\alpha_{1}=\alpha_{2}=0^{\circ}$ in the two-segment leg model with constant spring stiffness are found. Additionally, the range of landing angle becomes smaller with increased $\beta_{0}$; for example, in our model at $v_{0}=40 \mathrm{~m} \mathrm{~s}^{-1}$ and $\beta_{0}=115^{\circ}, \alpha_{1}=9.52^{\circ}$ and $\alpha_{2}=$ $7.62^{\circ}$ are found. However, at the same speed, both $\alpha_{1}$ and $\alpha_{2}$ at $\beta_{0}=165^{\circ}$ are only $0.95^{\circ}$. As for landing angle sensitivity, all three models are sensitive for angle of attack variations because of $\alpha_{1}=\alpha_{2}$, but our model is sensitive for angle of attack variations at higher speeds. Again, when our model is sensitive for landing angel variations, it is necessary to stable running at higher velocities for decreased $\beta_{0}$.

At fast running speed form 17 to $40 / 92 \mathrm{~m} \mathrm{~s}^{-1}$, regions of stable running of the proposed model are larger than those of the other two models, as illustrated in Figure 5(c). For instance, a region representing the number of successful running steps, shown in Figure 6, is made up of the $64 \times 64$ equidistant grid; for a given running speed of $29 \mathrm{~m} \mathrm{~s}^{-1}$ the stability region consists of the 247 equidistant grids in our model $\left(\beta_{0}=115^{\circ}\right)$. However, at the same running speed the region of stable running of the SLIP model is only the 16; worse still, there is no stable region in the two-segment leg model with constant spring stiffness. In contrast, at low running speed from 5 to $10 \mathrm{~m} \mathrm{~s}^{-1}$, our model demonstrates the minimum stability region in all three models. Again, in the two-segment leg model, an increase in nominal joint angle leads to a decrease of the stability region during high-speed locomotion. For instance, at high running speed $\left(36 \mathrm{~m} \mathrm{~s}^{-1}\right)$, the stability region of our model is decreased from the 205 at $\beta_{0}=115^{\circ}$ to the 10 equidistant grids at $\beta_{0}=165^{\circ}$.

3.4. Return Map of the Apex Height. In the following section we analyze stability of the proposed model by using of this single apex return map. For a given total energy $E=34424.8 \mathrm{~J}$ and system parameters $\left(\alpha_{0}=50^{\circ}\right.$ and $\left.\widetilde{k}_{10 \%}=18.75\right)$, results from the effects of different nominal joint angles on stable fixed points (the intersections of between three curves and the diagonal) are shown in Figure 7(a). From this figure, we can see that the small nominal joint angle results in the high value of fixed point. Here, all curves show that our model can accomplish periodic running patterns at the running speed of $29 \mathrm{~m} \mathrm{~s}^{-1}$.

Figure 7(b) shows the effects of another factor-different running velocities-on stable fixed points with system parameters $\left(\alpha_{0}=47^{\circ}, \widetilde{k}_{10 \%}=23.8\right.$, and $\left.\beta_{0}=115^{\circ}\right)$.

We can obtain that the lower the running speeds, the higher the values of fixed points, and the high running velocities $\left(31 \mathrm{~m} \mathrm{~s}^{-1}\right)$ result in the small basin of attraction containing all apex heights.

\section{Discussion}

In this paper, we discuss the effects of the two-segment leg with "J"-curve spring stiffness on running speeds during high-speed running. Two methods, the steps-to-fall analysis and the apex return map $[6,9]$, can be adopted to exploit high-speed running capacity of the model.

Compared with the other two models, during fast running it reveals that (1) system can provide the larger regions of stable running; (2) the tolerated range of $\widetilde{k}_{10 \%}$ for selfstable running is even larger for a given running speed; (3) the proposed model shows the larger tolerated speed range and running speeds. In addition, when the proposed model is stable running, the small nominal joint angle can lead to the low tolerated minimum $\widetilde{k}_{10 \%}$, with the large tolerated speed range and running speed. 


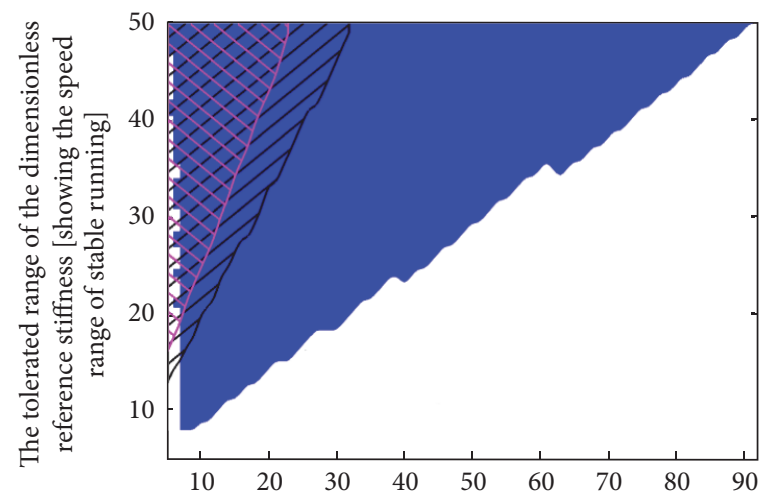

Running speed $v_{0}(\mathrm{~m} / \mathrm{s})$ two-segment leg model at $\beta_{0}=115^{\circ}$

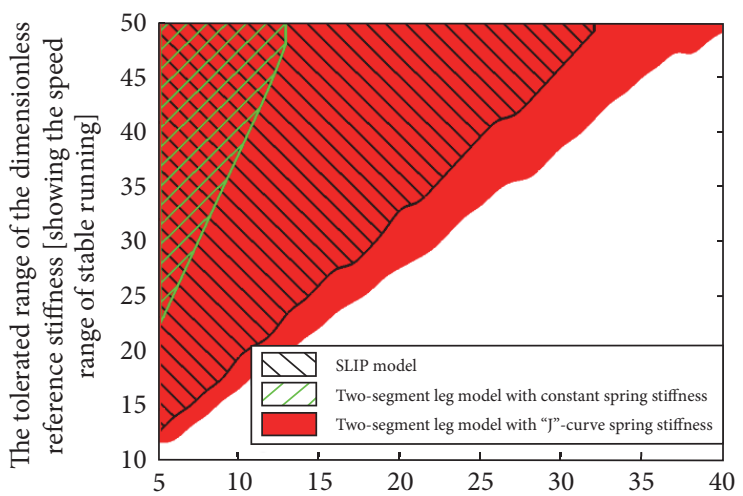

Running speed $v_{0}(\mathrm{~m} / \mathrm{s})$ two-segment leg model at $\beta_{0}=165^{\circ}$

77 SLIP model

$\square$ Two-segment leg model with constant spring stiffness

$\square$ Two-segment leg model with "J"-curve spring stiffness

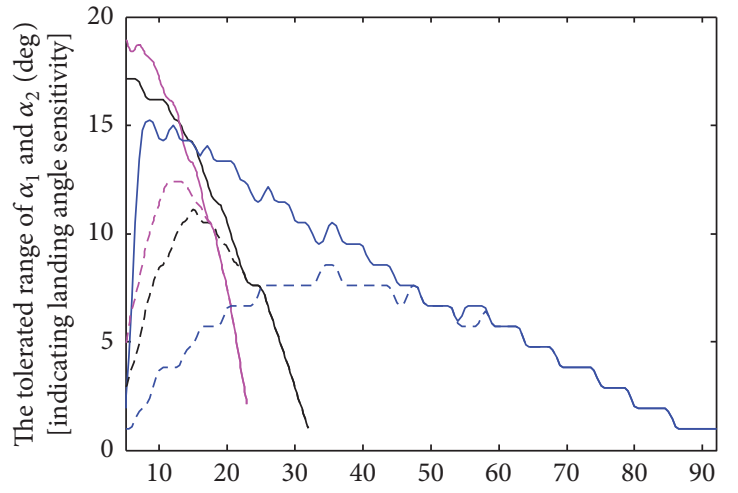

Running speed $v_{0}(\mathrm{~m} / \mathrm{s})$ two-segment leg model at $\beta_{0}=115^{\circ}$

$\alpha_{1}$ in the SLIP model

- $\alpha_{1}$ in the two-segment leg model with constant spring stiffness

$\alpha_{1}$ in the two-segment leg model with "J"-curve spring stiffness

$--\alpha_{2}$ in the SLIP model

- - $\alpha_{2}$ in the two-segment leg model with constant spring stiffness

- - $\alpha_{2}$ in the two-segment leg model with "J"-curve spring stiffness

(b)

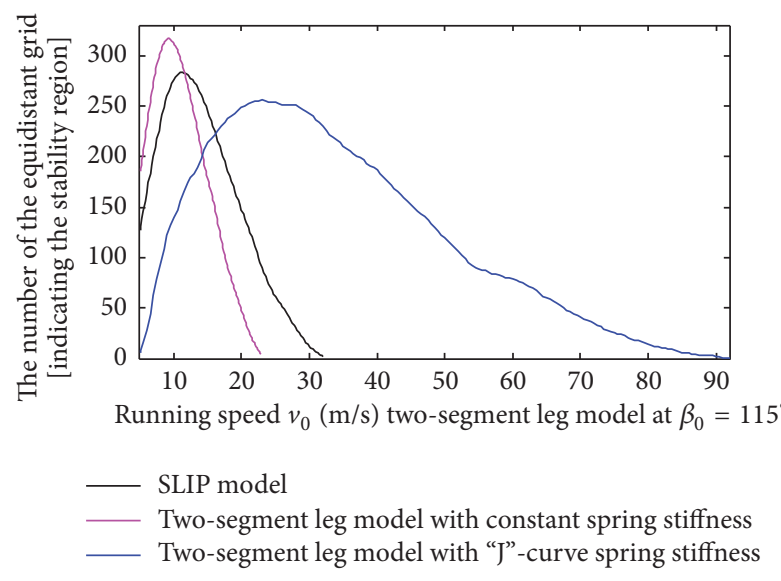

(a)

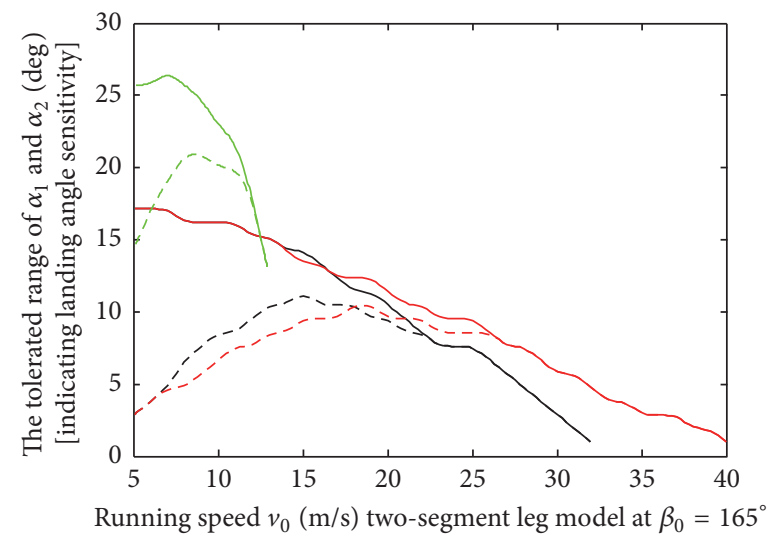

- $\alpha_{1}$ in the SLIP model

$\alpha_{1}$ in the two-segment leg model with constant spring stiffness

- $\alpha_{1}$ in the two-segment leg model with "J"-curve spring stiffness

- - $\alpha_{2}$ in the SLIP model

- - $\alpha_{2}$ in the two-segment leg model with constant spring stiffness

- - $\alpha_{2}$ in the two-segment leg model with "J"-curve spring stiffness

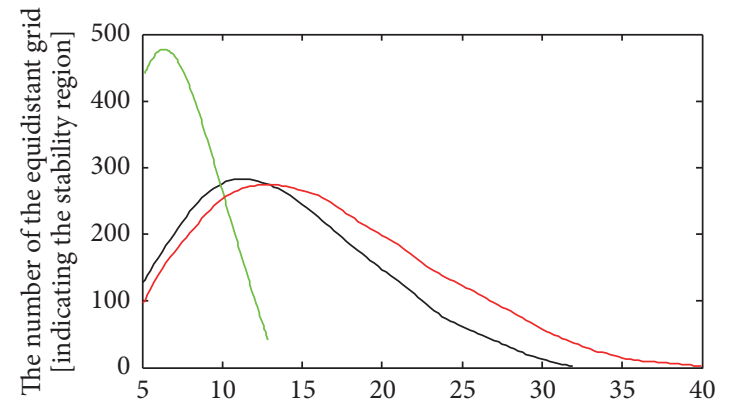

Running speed $v_{0}(\mathrm{~m} / \mathrm{s})$ two-segment leg model at $\beta_{0}=165^{\circ}$

_ SLIP model
Two-segment leg model with constant spring stiffness
Two-segment leg model with "J"-curve spring stiffness

(c)

Figure 5: Properties of regions of stable running for given combinations of $\widetilde{k}_{10 \%}$ and $\alpha_{0}$ at different running speeds in the SLIP model, the two-segment leg model with constant spring stiffness and with "J"-curve spring stiffness, respectively. Here, the speed range of stable running for a given $\widetilde{k}_{10 \%}$ from 0 to 50 is shown in (a) and (b), $\alpha_{1}$ denotes the difference between the minimum and maximum landing angles, and $\alpha_{2}$ is the maximum tolerated range of landing angle on a region of stable running. 


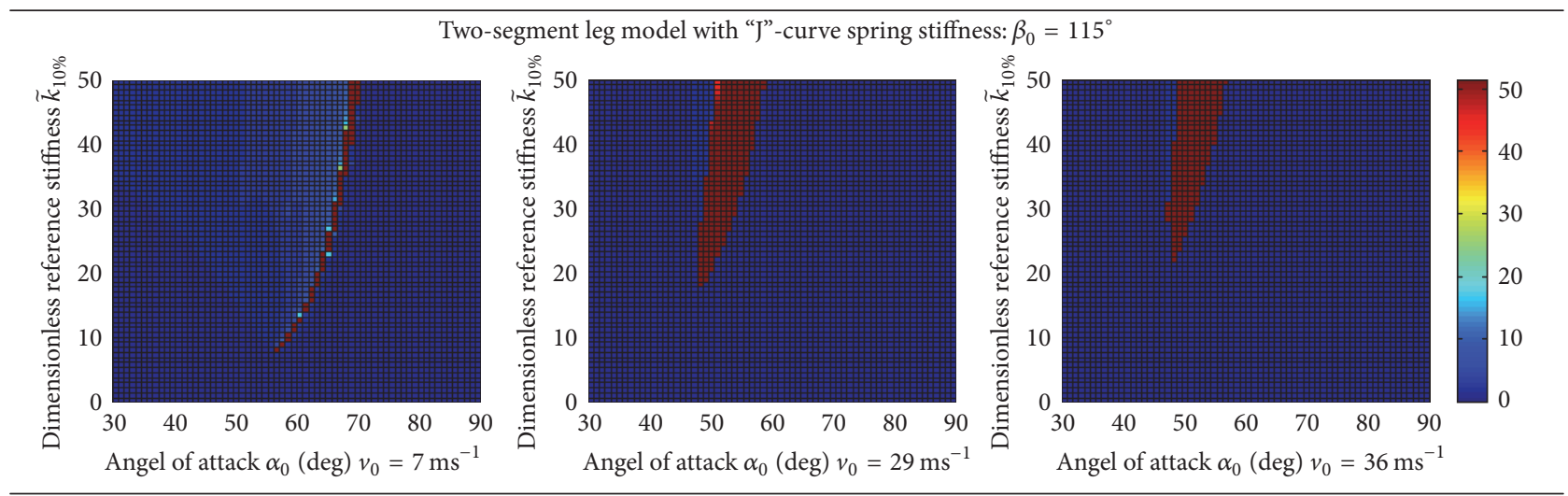

Two-segment leg model with "J"-curve spring stiffness: $\beta_{0}=165^{\circ}$
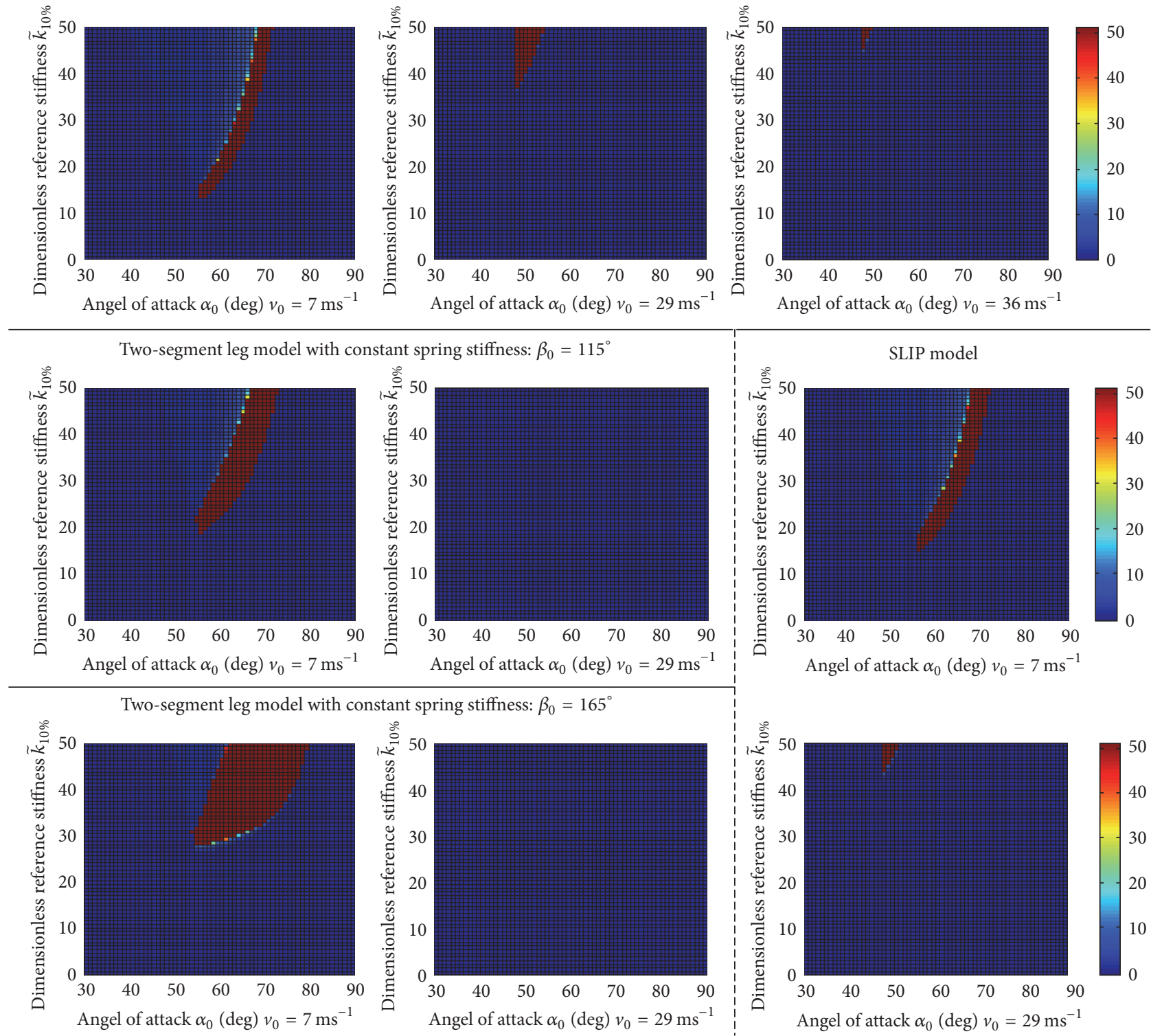

FIGURE 6: Regions of stable running for given combinations of $\widetilde{k}_{10 \%}$ and $\alpha_{0}$ in the two-segment leg model with "J"-curve spring stiffness (the first row $\beta_{0}=115^{\circ}$ and the second row $\beta_{0}=165^{\circ}$ ), the two-segment leg model with constant spring stiffness (the first and second figures of the third row $\beta_{0}=115^{\circ}$ and the first and second figures of the last row $\beta_{0}=165^{\circ}$ ) and the SLIP model (the third and fourth figures of the third column). 


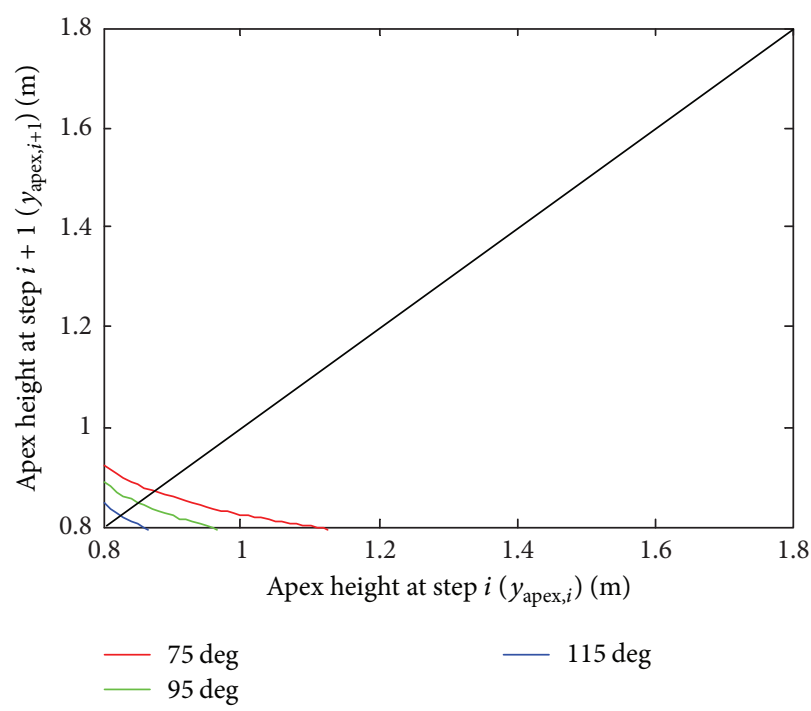

(a) Return maps function $y_{\text {apex }, i+1}\left(y_{\text {apex }, i}\right)$ at different $\beta_{0}$

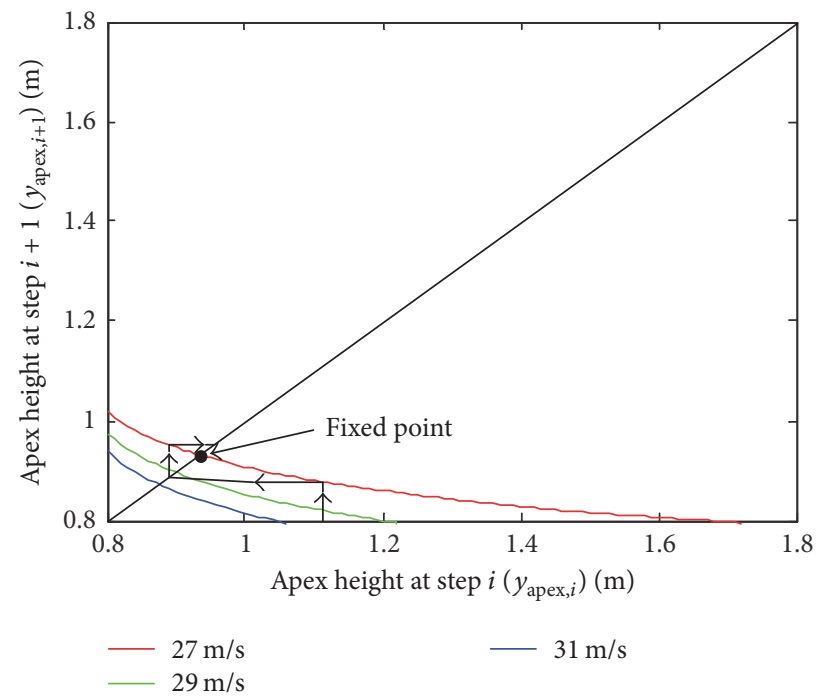

(b) Return maps function $y_{\text {apex }, i+1}\left(y_{\text {apex }, i}\right)$ at different $v_{0}$

FIGURE 7: Return maps of the apex height $y_{\text {apex }, i+1}\left(y_{\text {apex }, i}\right)$ of a single step in the two-segment leg model with "J"-curve spring stiffness.

These characteristics mentioned above are due to the two-segment leg structural configuration and "J"-curve spring stiffness properties, resulting in the nonlinear forcecompression relationships depicted in Figure 4. In addition, owing to the passive compliance identified in the proposed model, this elastic two-segment leg configuration can take advantage of simple control strategies to guarantee the steadystate running behavior, with little or even no sensory information. We believe that our model can be seen as a template to analyze the high-speed dynamic locomotion for animals and robots. However, this two-segment leg model with a "J"-shape force-elongation curve cannot mimic adjustment of leg stiffness in fast animal locomotion. This is because limb stiffness is adapted to running speed; for example, Hobara et al. [28] reveal through experiments that in humans leg stiffness is adjusted to different hopping frequencies, with the fact that there are two different force-elongation curves during compression and decompression. Hence, in order to adequately mimic limb compliant locomotion, these aspects should be taken into account in the design and implementation of robotic system.

Additionally, in the two-segment leg model with "J"curve spring stiffness, the running is simulated across smooth and level terrain. Yet, the terrain is not the same in the real world, where the ground surface irregularities must be taken into account for running robots and running models. Therefore, in order to achieve stable running of the proposed model on uneven terrain, it is necessary to adjust apex height with adequate ground clearance in response to disturbances in ground height. Currently, for a given total system energy, the return map of the apex height $y_{\text {apex }, i+1}\left(y_{\text {apex }, i}\right)$ can be adopted to analyze running stability when the system is perturbed by a change in ground height. Furthermore, as the proposed model is conservative, the total system energy is distributed to the vertical energy and the forward kinetic energy by the adjustment of angle of attack; in other words,

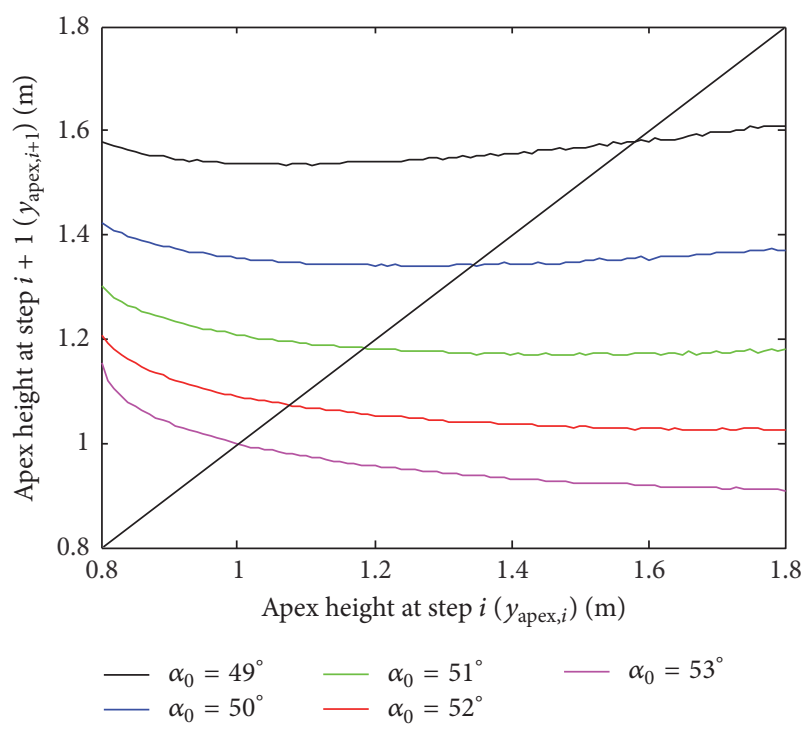

FIGURE 8: Return maps function $y_{\text {apex }, i+1}\left(y_{\text {apex }, i}\right)$ at different $\alpha_{0}$.

different angles of attack can be used to control a desired apex height or, correspondingly, the forward speed. Here, the return map of the apex height is adopted to investigate the potential effects of different landing angles on the apex height in our model $\left(v_{0}=29 \mathrm{~m} \mathrm{~s}^{-1}, \widetilde{k}_{10 \%}=40\right.$, and $\beta_{0}=$ $\left.115^{\circ}\right)$. Results shown in Figure 8 reveal that the apex height $y_{\text {apex }, i+1}$ is dependent on the preceding apex height $y_{\text {apex }, i}$ and the selected angle of attack $\alpha_{0}$. What is more, for a given apex height $y_{\text {apex }, i}$, both the value of fixed point and the subsequent apex height $y_{\text {apex }, i+1}$ become increasingly small with increasing $\alpha_{0}$, and the apex height is sensitive for angle of attack variations. This means that a higher apex height can be implemented by tuning a smaller angle of attack, with the fact that only one step or a few more steps are 
required to achieve higher steady-state heights in our model. Again, it is interesting to note that Seyfarth and Geyer [29] introduce a generalized return map and derive an optimal control strategy of the apex height in the SLIP model. This method could be utilized to study the control strategy of the apex height of the proposed model, which will be subject of further investigations.

Running at High Speed. In Figure 5(a), the SLIP model indicates that the tolerated minimum leg stiffness has to increase with running speed to achieve stable running. This is in agreement with a simulation study in which increasing leg stiffness is required to guarantee the stability of a galloping quadruped whose single leg consists of a linear spring attached to a prismatic leg when running speed is increasingly high [30]. In the two-segment leg model with constant spring stiffness, an increase in the tolerated minimum leg stiffness is also necessary to accomplish stable running behavior at higher speeds [6]. This finding is supported by robotic trials. Here, hopping robot with one leg, which is made up of a segmented leg with constant spring stiffness, becomes unstable with increasing speed [31]. Therefore, leg stiffness is sensitive for speed variation in these two models. The situation is not all the same in the two-segment leg model with "J"curve spring stiffness. Here, in our model the amount of increment in the tolerated minimum dimensionless reference stiffness, for the same amount of increment in running speed, is largely decreased compared with the other two models. This characteristic of our model (leg stiffness is insensitive for speed variation) enlarges tolerated range of speeds. On the other hand, for a given running speed, the proposed model has the minimum value of leg stiffness to achieve selfstable running in all three models, as depicted in Figure 5(a). For instance, at high running speed $\left(29 \mathrm{~m} \mathrm{~s}^{-1}\right)$, the tolerated minimum dimensionless reference stiffness in our model at $\beta_{0}=115^{\circ}$ is 18.25 compared with 44.44 in linear leg model and no stability solutions in the two-segment leg model with constant spring stiffness. As a result, in all three models, for a given nominal joint angle and leg stiffness, the proposed model at high speed has not only the maximum speed range of stable locomotion but also the tolerated maximum running speed. This means that in all three models the twosegment leg model with "J"-curve spring stiffness is most advantageous for high-speed running.

Practically, several researchers have designed their robotic legs similar to our model configuration and leg stiffness. For example, the robotic leg proposed by Schmiedeler and Waldron [32] used seven parameters to accomplish biomimetic feature. With these parameters, we here in this paper have calculated their virtual leg force-compression relationship, as shown in Figure9. Obviously, this curve is similar to the curves as represented by our model depicted in Figure 4, leading to the following common characteristics: (1) the virtual leg spring is capable of being soft at the beginning of touch down, which can produce a relatively small impact force [32]; (2) it becomes stiffer with increased compression, resulting in a large force, which can prevent the leg to fold over on itself [32]. With the facts that their prototype leg exhibits the potential for fast

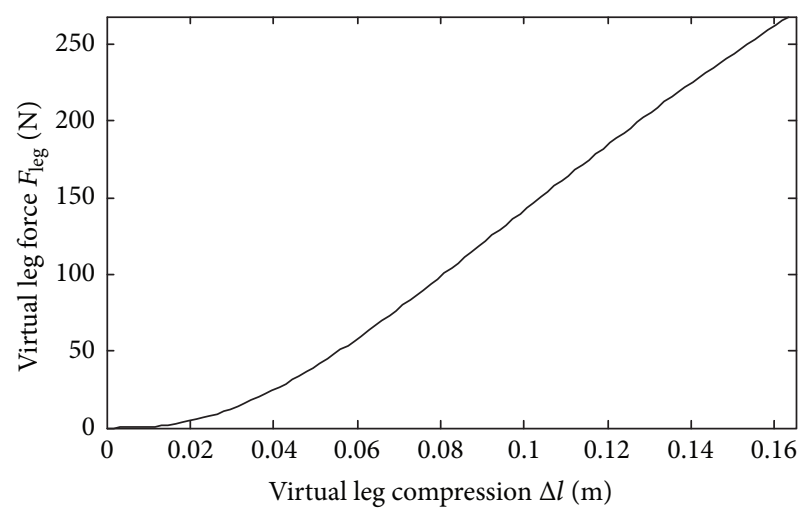

Figure 9: Virtual leg force-compression relationships of KOLT quadruped robot.

locomotion in experiment [32] and then the corresponding KOLT quadruped robot can implement gallop and highspeed turning [33], we conclude that our simulation results have been confirmed in practice.

\section{Conclusion}

In this paper, we have presented the two-segment leg model with "J"-curve spring stiffness and have analyzed the effects of "J"-curve spring stiffness on running speeds of the proposed model during high-speed running. According to simulation results of all three models, for a given dimensionless reference stiffness, we have demonstrated that (1) our model has not only the largest tolerated speed range but also the tolerated maximum running speed, with the fact that the smaller the nominal joint angle, the better the running capacity mentioned above; (2) at fast running speed from 25 to $40 / 92 \mathrm{~m} \mathrm{~s}^{-1}$ our model has not only the largest range of angle of attack but the largest region of stable running. It has already been successfully applied to quadruped robot, such as KOLT, for high-speed running.

Next, we will investigate the effects of a broad ratio of leg length and nominal joint angle range on high-speed running performance, respectively. Again, we will analyze the whole stability zone of segmented leg in order to gain better insights into advantages and disadvantages of our model at fast running.

\section{Competing Interests}

The authors declare that there is no conflict of interests regarding the publication of this paper.

\section{Acknowledgments}

This project is supported by National Natural Science Foundation of China (Grant no. 51475373).

\section{References}

[1] R. M. Alexander, "Tendon elasticity and muscle function," Comparative Biochemistry and Physiology: A Molecular and Integrative Physiology, vol. 133, no. 4, pp. 1001-1011, 2002. 
[2] Y. Blum, S. W. Lipfert, J. Rummel, and A. Seyfarth, "Swing leg control in human running," Bioinspiration and Biomimetics, vol. 5, no. 2, Article ID 026006, 2010.

[3] R. M. Alexander, "Three uses for springs in legged locomotion," The International Journal of Robotics Research, vol. 9, no. 2, pp. 53-61, 1990.

[4] A. Seyfarth, H. Geyer, and H. Herr, "Swing-leg retraction: a simple control model for stable running," Journal of Experimental Biology, vol. 206, no. 15, pp. 2547-2555, 2003.

[5] A. A. Biewener, Animal Locomotion, Oxford University Press, Oxford, UK, 2003.

[6] J. Rummel and A. Seyfarth, "Stable running with segmented legs," International Journal of Robotics Research, vol. 27, no. 8, pp. 919-934, 2008.

[7] M. F. Bobbert and L. J. R. Casius, "Spring-like leg behaviour, musculoskeletal mechanics and control in maximum and submaximum height human hopping," Philosophical Transactions of the Royal Society B: Biological Sciences, vol. 366, no. 1570, pp. 1516-1529, 2011.

[8] R. M. Alexander and A. S. Jayes, "Vertical movement in walking and running," Journal of Zoology, vol. 185, no. 1, pp. 27-40, 1978.

[9] A. Seyfarth, H. Geyer, M. Günther, and R. Blickhan, "A movement criterion for running," Journal of Biomechanics, vol. 35, no. 5, pp. 649-655, 2002.

[10] A. Sato and M. Buehler, "A planar hopping robot with one actuator: design, simulation, and experimental results," in Proceedings of the 2004 IEEE/RSJ International Conference on Intelligent Robots and Systems (IROS '04), pp. 3540-3545, Sendai, Japan, October 2004.

[11] A. Seyfarth, M. Günther, and R. Blickhan, "Stable operation of an elastic three-segment leg," Biological Cybernetics, vol. 84, no. 5, pp. 365-382, 2001.

[12] A. H. Hansen, D. S. Childress, S. C. Miff, S. A. Gard, and K. P. Mesplay, "The human ankle during walking: implications for design of biomimetic ankle prostheses," Journal of Biomechanics, vol. 37, no. 10, pp. 1467-1474, 2004.

[13] J. Rummel, F. Iida, J. A. Smith, and A. Seyfarth, "Enlarging regions of stable running with segmented legs," in Proceedings of the IEEE International Conference on Robotics and Automation (ICRA '08), pp. 367-372, Barcelona, Spain, May 2008.

[14] J. D. Karssen and M. Wisse, "Running with improved disturbance rejection by using non-linear leg springs," The International Journal of Robotics Research, vol. 30, no. 13, pp. 1585-1595, 2011.

[15] K. Sreenath, H.-W. Park, I. Poulakakis, and J. W. Grizzle, "Embedding active force control within the compliant hybrid zero dynamics to achieve stable, fast running on MABEL," The International Journal of Robotics Research, vol. 32, no. 3, pp. 324345, 2013.

[16] A. Spröwitz, A. Tuleu, M. Vespignani, M. Ajallooeian, E. Badri, and A. J. Ijspeert, "Towards dynamic trot gait locomotion: design, control, and experiments with Cheetah-cub, a compliant quadruped robot," The International Journal of Robotics Research, vol. 32, no. 8, pp. 932-950, 2013.

[17] K. Sreenath, H.-W. Park, and J. W. Grizzle, "Design and experimental implementation of a compliant hybrid zero dynamics controller with active force control for running on MABEL," in Proceedings of the IEEE International Conference on Robotics and Automation (ICRA '12), pp. 51-56, St Paul, Minn, USA, May 2012.
[18] C. V. Jutte and S. Kota, "Design of nonlinear springs for prescribed load-displacement functions," Journal of Mechanical Design, Transactions of the ASME, vol. 130, no. 8, Article ID 081403, 2008.

[19] R. M. Alexander, "Elastic energy stores in running vertebrates," American Zoologist, vol. 24, no. 1, pp. 85-94, 1984.

[20] H. Zhao, Y.-N. Wu, M. Hwang et al., "Changes of calf muscletendon biomechanical properties induced by passive-stretching and active-movement training in children with cerebral palsy," Journal of Applied Physiology, vol. 111, no. 2, pp. 435-442, 2011.

[21] S. Fukashiro, M. Rob, Y. Ichinose, Y. Kawakami, and T. Fukunaga, "Ultrasonography gives directly but noninvasively elastic characteristic of human tendon in vivo," European Journal of Applied Physiology and Occupational Physiology, vol. 71, no. 6, pp. 555-557, 1995.

[22] D. A. Winter, A. E. Patla, S. Rietdyk, and M. G. Ishac, "Ankle muscle stiffness in the control of balance during quiet standing," Journal of Neurophysiology, vol. 85, no. 6, pp. 2630-2633, 2001.

[23] S. Vogel, Cats' Paws and Catapults, W. W. Norton, New York, NY, USA, 1998.

[24] R. Blickhan and R. J. Full, "Similarity in multilegged locomotion: bouncing like a monopode," Journal of Comparative Physiology A, vol. 173, no. 5, pp. 509-517, 1993.

[25] C. T. Farley and O. González, "Leg stiffness and stride frequency in human running," Journal of Biomechanics, vol. 29, no. 2, pp. 181-186, 1996.

[26] H. Geyer, A. Seyfarth, and R. Blickhan, "Spring-mass running: simple approximate solution and application to gait stability," Journal of Theoretical Biology, vol. 232, no. 3, pp. 315-328, 2005.

[27] N. C. C. Sharp, "Timed running speed of a cheetah (Acinonyx jubatus)," Journal of Zoology, vol. 241, no. 3, pp. 493-494, 1997.

[28] H. Hobara, Y. Kobayashi, E. Yoshida, and M. Mochimaru, "Leg stiffness of older and younger individuals over a range of hopping frequencies," Journal of Electromyography and Kinesiology, vol. 25, no. 2, pp. 305-309, 2015.

[29] A. Seyfarth and H. Geyer, "Natural control of spring-like running: optimized selfstabilization," in Proceedings of the 5th International Conference on Climbing and Walking Robots (CLAWAR '02), pp. 81-85, September 2002.

[30] P. Nanua and K. J. Waldron, "Instability and chaos in quadruped gallop," Journal of Mechanical Design, Transactions Of the ASME, vol. 116, no. 4, pp. 1096-1101, 1994.

[31] J. Rummel, F. Iida, and A. Seyfarth, "One-legged locomotion with a compliant passive joint," in Proceedings of the 9th International Conference on Intelligent Autonomous Systems (IAS '06), pp. 566-573, Tokyo, Japan, March 2006.

[32] J. P. Schmiedeler and K. J. Waldron, "Leg stiffness and articulated leg design for dynamic locomotion," in Proceedings of the ASME International Design Engineering Technical Conferences, pp. 1105-1112, September 2002.

[33] L. R. Palmer III and D. E. Orin, "Intelligent control of highspeed turning in a quadruped," Journal of Intelligent and Robotic Systems: Theory and Applications, vol. 58, no. 1, pp. 47-68, 2010. 


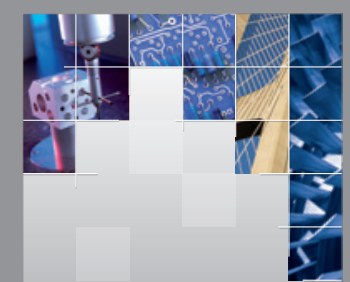

\section{Enfincering}
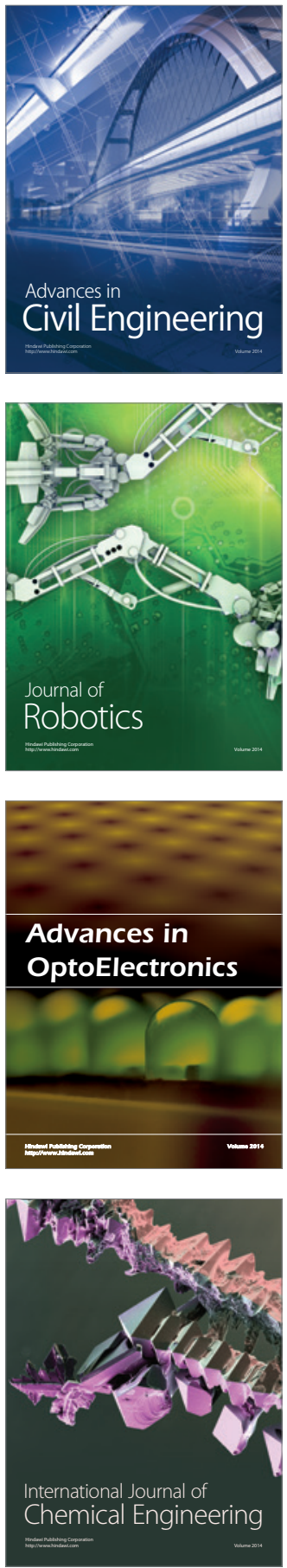

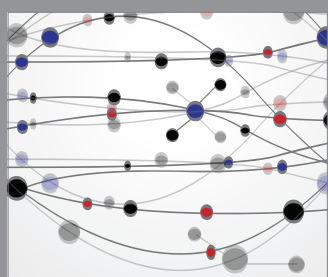

The Scientific World Journal

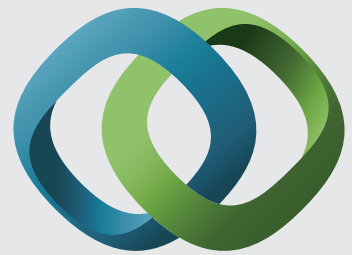

\section{Hindawi}

Submit your manuscripts at

http://www.hindawi.com
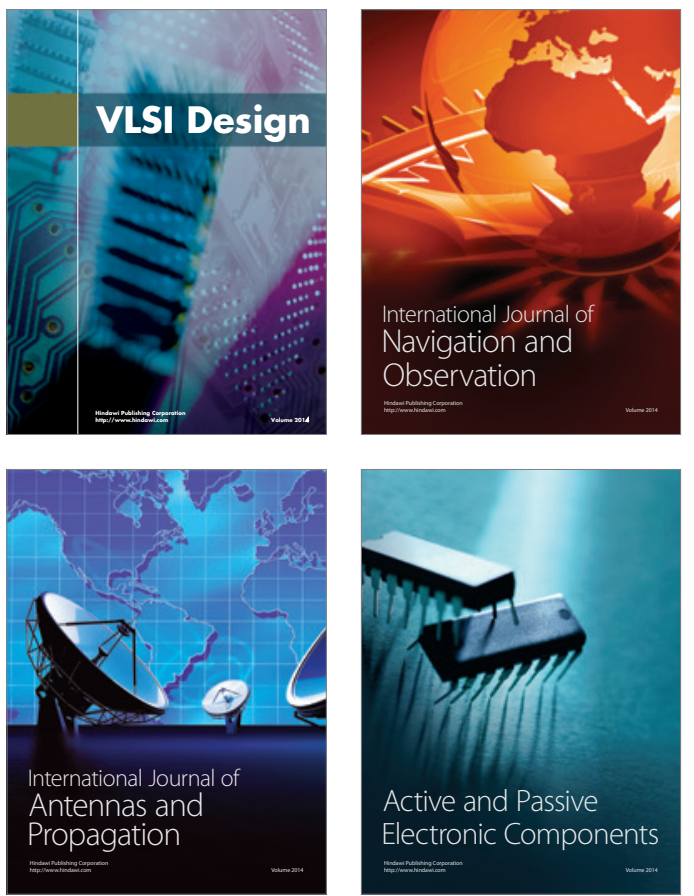
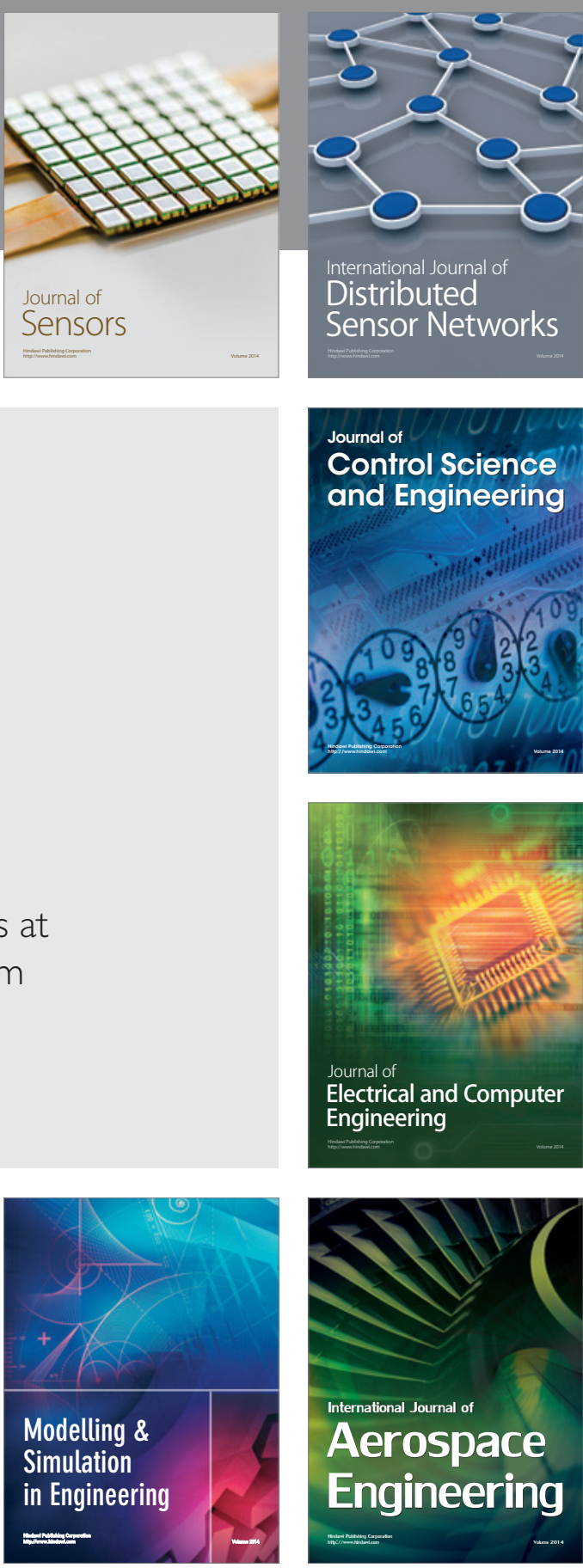

International Journal of

Distributed

Sensor Networks

Journal of

Control Science

and Engineering
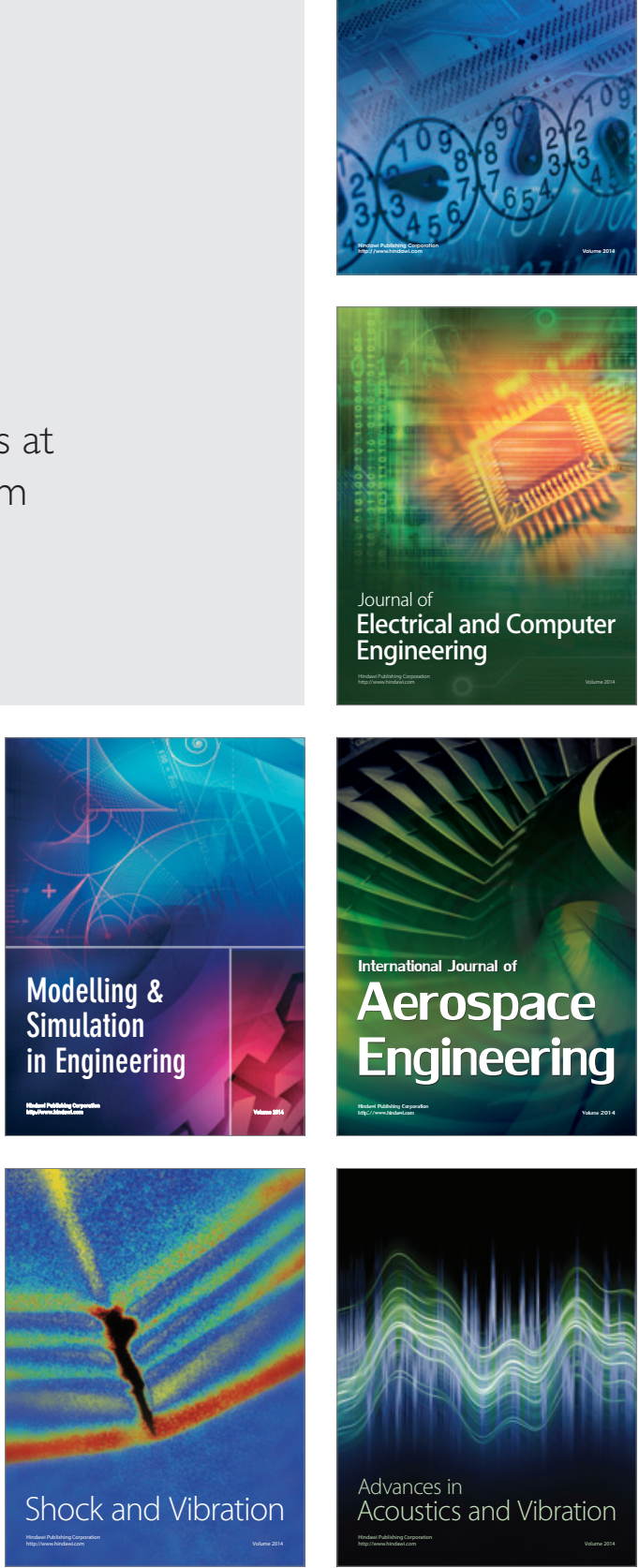